\title{
Fasting and Postprandial Activity of Brown Adipose Tissue in Healthy Men
}

\author{
Anne Vrieze ${ }^{1}$, Josefine E. Schopman ${ }^{1}$, Wanda M. Admiraal ${ }^{1}$, Maarten R. Soeters ${ }^{2}$, Max Nieuwdorp ${ }^{1,2}$, Hein J. Verberne ${ }^{3}$, \\ and Frits Holleman ${ }^{1}$ \\ ${ }^{1}$ Department of Internal Medicine, Academic Medical Center, Amsterdam, The Netherlands; ${ }^{2}$ Department of Endocrinology and \\ Metabolism, Academic Medical Center, Amsterdam, The Netherlands; and ${ }^{3}$ Department of Nuclear Medicine, Academic Medical \\ Center, Amsterdam, The Netherlands
}

\begin{abstract}
The role of brown adipose tissue (BAT) in adult metabolism is poorly understood. This study aimed to examine the differential effects of an overnight fast and the postprandial state on BAT activity. Methods: We included 10 healthy, lean male volunteers. BAT uptake of glucose was visualized using ${ }^{18} \mathrm{~F}-\mathrm{FDG}$ PET/CT during mild cold exposure. Each subject underwent PET/CT twice. The first scan was obtained after an overnight fast; the second after a standardized meal. Results: ${ }^{18} \mathrm{~F}-\mathrm{FDG}$ uptake in BAT was observed in 6 of 10 volunteers. These subjects were found to have a higher maximal standardized uptake value when fasting (median, $13.1 \mathrm{~g} / \mathrm{mL}$; range, 6.1-27.6 $\mathrm{g} / \mathrm{mL}$ ) than when in the postprandial state (median, $6.8 \mathrm{~g} / \mathrm{mL}$; range, $2.1-13.4 \mathrm{~g} / \mathrm{mL})(P=0.03)$. Conclusion: Cold-stimulated ${ }^{18} \mathrm{~F}-$ FDG uptake by BAT in humans is more pronounced during fasting. The lower maximal standardized uptake value in the postprandial state may be explained by increased insulin-stimulated glucose uptake in muscle.
\end{abstract}

Key Words: BAT; ${ }^{18}$ F-FDG uptake; glucose metabolism

J Nucl Med 2012; 53:1407-1410

DOI: 10.2967/jnumed.111.100701

B rown adipose tissue (BAT), long thought to dwindle after infancy, is present and physiologically active in many adults (1-3). When BAT is activated, it initiates a series of pathways that convert free fatty acids and glucose into heat, rather than storing these. Harnessing BAT to increase energy expenditure in humans might be a novel approach to fight obesity (4).

Long before BAT drew the attention of the wider medical community, specialists recognized that ${ }^{18} \mathrm{~F}-\mathrm{FDG}$ PET scans used for clinical practice were best obtained under warm and fasting conditions to avoid false-positive findings. The introduction of PET/CT further reduced the

Received Nov. 28, 2011; revision accepted May 9, 2012.

For correspondence or reprints contact: Anne Vrieze, Department of Internal Medicine, Academic Medical Center, Meibergdreef 9, Room F4-

256, 1105 AZ Amsterdam, the Netherlands.

E-mail: a.vrieze@amc.uva.nl

Published online Jul. 31, 2012.

COPYRIGHT (C 2012 by the Society of Nuclear Medicine and Molecular Imaging, Inc. false-positive rate and could explain some of the falsepositives as attributable to ${ }^{18} \mathrm{~F}-\mathrm{FDG}$ uptake by BAT.

BAT in adults can be visualized on PET/CT using ${ }^{18} \mathrm{~F}$ FDG (1-3) under conditions of mild cold exposure. BAT activity is significantly influenced by body mass index, sex, and temperature (5). In rats, besides mild cold exposure, feeding with concomitantly elevated plasma insulin levels leads to increased uptake of glucose by BAT $(6,7)$. Conversely, in the setting of low insulin levels or insulin resistance, this uptake is reduced $(6,7)$.

A recent study on healthy humans suggested that intravenous insulin, in a warm environment, stimulates glucose uptake by BAT (8). The importance of diet-induced thermogenesis in humans is subject to debate, and data on human BAT in the postprandial state are limited. Moreover, the importance of lipids as a substrate for BAT warrants the investigation of BAT activity after a high-fat meal (9). Therefore, the aim of our study was to examine ${ }^{18} \mathrm{~F}-\mathrm{FDG}$ glucose uptake in BAT during cold exposure in the fasting and postprandial states.

\section{MATERIALS AND METHODS}

We studied a group of 10 healthy, lean male volunteers (18$32 \mathrm{y}$; body mass index, $20-24 \mathrm{~kg} / \mathrm{m}^{2}$ ). The institutional ethics committee approved the protocol, and written informed consent was obtained from all subjects. Subjects were recruited through public advertisements.

Each of the 10 subjects underwent 2 PET/CT scans with ${ }^{18} \mathrm{~F}-\mathrm{FDG}$, separated by at least $2 \mathrm{wk}$. The first scan was obtained after an overnight fast; the second after an overnight fast, with a standardized meal taken $90 \mathrm{~min}$ before ${ }^{18} \mathrm{~F}-\mathrm{FDG}$ administration (chicken-bacon sandwich and $200 \mathrm{~mL}$ of whole milk (545 kcal), containing $34 \mathrm{~g}$ of fat, $37 \mathrm{~g}$ of carbohydrates, and $23 \mathrm{~g}$ of protein [respectively, 56\%, 27\%, and $17 \%$ of the energy]).

\section{Scanning Protocol}

Subjects were exposed to mild cold $\left(16^{\circ} \mathrm{C}-18^{\circ} \mathrm{C}\right.$, controlled by use of a ventilation system [Airco]) for $2 \mathrm{~h}$ in both experiments to avoid confounding effects from the presence or absence of cold. Shivering was neither reported by subjects nor noticed by the research staff. After $1 \mathrm{~h}$ of exposure to cold, approximately $200 \mathrm{MBq}$ of ${ }^{18} \mathrm{~F}-\mathrm{FDG}$ were administered intravenously, 
and exposure to cold was continued for a second hour. Upperbody (from the base of the skull to the groin) static PET was performed $60 \mathrm{~min}$ after ${ }^{18} \mathrm{~F}$-FDG injection. All scanning was performed in the cold season (between December and February).

For both fasting and postprandial PET/CT, baseline fasting blood samples were drawn for the determination of glucose and insulin levels. For postprandial PET/CT, blood samples were also taken 90 min after the meal to determine glucose and insulin levels; ${ }^{18} \mathrm{~F}$-FDG administration followed immediately.

PET/CT images were acquired with a Gemini time-of-flight multidetector helical PET/CT scanner (Philips). In areas where uptake of ${ }^{18} \mathrm{~F}$-FDG was identified by PET and the presence of fat was identified by CT (Hounsfield units between -250 and -50 ), the maximal standardized uptake value (SUVmax) - defined as the activity in becquerels per milliliter within the region of interest divided by the injected dose in becquerels per gram of body weight - was determined (Hybrid Viewer; HERMES Medical Solutions). Anatomic regions of interest were the cervical, supraclavicular, and superior mediastinal depots. In these areas, an SUVmax of at least $2.0 \mathrm{~g} / \mathrm{mL}$ was considered to indicate BAT (1).

\section{Statistical Analysis}

Data are reported as median with range. Data analysis was performed with SPSS software, version 16 (IBM). The ${ }^{18} \mathrm{~F}-\mathrm{FDG}$ uptake in the fasting state was compared with the ${ }^{18} \mathrm{~F}-\mathrm{FDG}$ uptake in the postprandial state by means of the nonparametric Wilcoxon signed-rank test. $P$ values of less than 0.05 were considered statistically significant.

\section{RESULTS}

${ }^{18} \mathrm{~F}-\mathrm{FDG}$ uptake in BAT was observed in 6 of 10 volunteers. Table 1 shows the characteristics of the 10 volunteers, categorized as those with detectable BAT and those with undetectable BAT. There was no significant difference in clinical characteristics between the 2 groups. All subjects with ${ }^{18}$ F-FDG uptake in BAT had a higher SUVmax in the fasting state (median, $13.1 \mathrm{~g} / \mathrm{mL}$; range, 6.1-27.6 g/mL) than in the postprandial state (median, $6.8 \mathrm{~g} / \mathrm{mL}$; range,
$2.1-13.4 \mathrm{~g} / \mathrm{mL})(P=0.03$, Fig. 1$)$. In addition, in the postprandial state substantial ${ }^{18} \mathrm{~F}-\mathrm{FDG}$ uptake in skeletal muscles was noted, whereas in the fasting state hardly any ${ }^{18}$ F-FDG muscle uptake was visible (Fig. 2). The median SUVmax in skeletal muscles was $2.0 \mathrm{~g} / \mathrm{mL}$ (range, $0.8-2.2 \mathrm{~g} / \mathrm{mL}$ ) in the postprandial state, compared with $0.8 \mathrm{~g} / \mathrm{mL}$ (range, $0.8-0.9 \mathrm{~g} / \mathrm{mL})$ in the fasting state $(P=$ 0.03). If we divide the SUVmax of BAT by the SUVmax of muscle, we find that BAT metabolic activity was 15-fold higher than muscle activity after fasting and 4-fold higher after the meal $(P=0.03)$.

\section{DISCUSSION}

Physiologically active BAT was found in $60 \%$ of the subjects at a room temperature of $16^{\circ} \mathrm{C}-18^{\circ} \mathrm{C}$. Although more subjects would probably have shown BAT uptake if the temperature had been lower, a lower temperature would probably have caused shivering, thus making our assessment of nonshivering thermogenesis less reliable. Most studies performed to date have used similar temperatures, and the prevalence of $60 \%$ is in line with the literature (2).

In contrast to results found in rats, the present study showed that glucose uptake by BAT in humans is more pronounced in the fasting state. The different biodistribution pattern of ${ }^{18} \mathrm{~F}-\mathrm{FDG}$ in the postprandial state may be explained by increased ${ }^{18}$ F-FDG retention by muscle because of the meal-induced insulin secretion, thus reducing the availability of ${ }^{18} \mathrm{~F}-\mathrm{FDG}$ for uptake by BAT

In contrast, Orava et al. found an increase in BAT uptake of glucose after insulin stimulation. This finding might be attributed to the fact that they used arterial ${ }^{18} \mathrm{~F}$ FDG sampling and dynamic scanning to estimate ${ }^{18} \mathrm{~F}-\mathrm{FDG}$ uptake - a method that yields uptake rates per minutewhereas our method yields the cumulative uptake in the first hour (no absolute qualification was performed). How-

TABLE 1

Characteristics of Volunteers $(n=10)$

\begin{tabular}{lrr}
\hline \multicolumn{1}{c}{ Characteristic } & BAT $+(n=6)$ & BAT- $(n=4)$ \\
\hline Age $(\mathrm{y})$ & $24.5(18-32)$ & $25.5(21-29)$ \\
Body mass index $\left(\mathrm{kg} / \mathrm{m}^{2}\right)$ & $22.2(20.3-24.0)$ & $22.2(20.9-23.4)$ \\
\hline First study day & $5.2(4.1-5.4)$ & $4.7(3.6-4.7)$ \\
$\quad$ Fasting plasma glucose $(\mathrm{mmol} / \mathrm{L})$ & $23.5(15-52)$ & $15.0(15-15)$ \\
$\quad$ Fasting plasma insulin $(\mathrm{pmol} / \mathrm{L})$ & $4.7(4.6-5.3)$ & $4.6(4.5-4.8)$ \\
\hline Second study day & $15.5(15-44)$ & $15.0(15-16)$ \\
$\quad$ Fasting plasma glucose $(\mathrm{mmol} / \mathrm{L})$ & $4.3(2.6-4.9)$ & $4.1(3.7-5.1)$ \\
$\quad$ Fasting plasma insulin $(\mathrm{pmol} / \mathrm{L})$ & $116.5(51-169)$ & $43.0(15-128)$ \\
$\quad$ Plasma glucose after meal $(\mathrm{mmol} / \mathrm{L})$ & & \\
$\quad$ Plasma insulin after meal $(\mathrm{pmol} / \mathrm{L})$ & & \\
& & \\
\hline BAT+ $=$ detectable; BAT- $=$ undetectable. & & \\
Data are median, followed by range in parentheses. & & \\
\hline
\end{tabular}




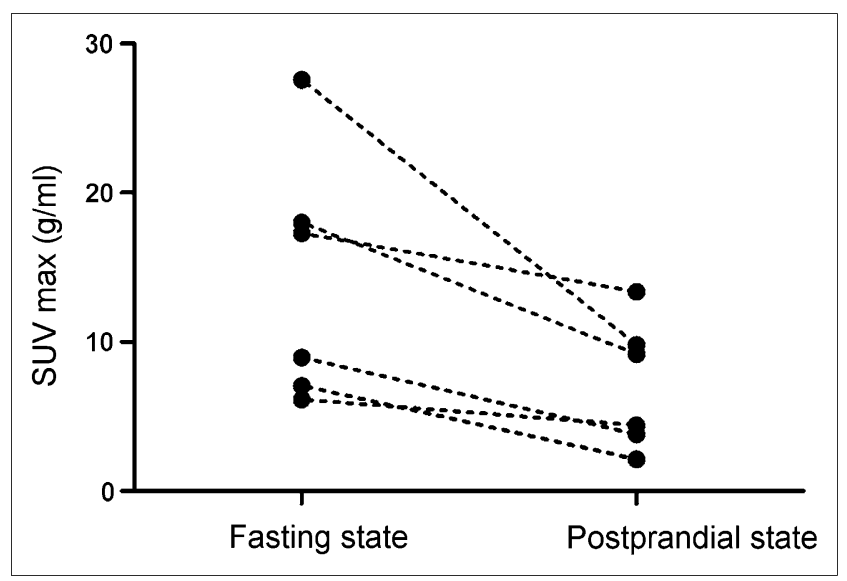

FIGURE 1. Overview of change in BAT for each volunteer. BAT activity is reduced in postprandial state in all volunteers with detectable BAT.

ever, comparison of the cumulative uptake in the first hour should be a reliable indicator of total activity since uptake is still ongoing at that time. Moreover, Orava et al. studied subjects in a warm environment and found an approximately 5-fold increase after insulin in cold, and in fasting subjects they found an approximately 12 -fold increase in ${ }^{18} \mathrm{~F}$-FDG uptake, which supports the notion that insulin is not the principal determinant of BAT activity (8). Finally, in contrast to the controlled intervention of a hyperinsulinemic euglycemic clamp, postprandial BAT metabolism and energy expenditure are under the control of a complicated physiologic response to the meal. Multiple mechanisms may affect energy expenditure, such as diet-induced thermogenesis and the thermic effect of food itself (e.g., metabolic, endocrine, cardiovascular, sympathetic, and gut responses) (10).

Another explanation for the lower glucose uptake in the postprandial state might be a relatively higher contribution of lipid oxidation to total BAT energy expenditure. Although ${ }^{18} \mathrm{~F}-\mathrm{FDG}$ uptake has been considered and shown to be a good reflection of BAT activity in most studies performed to date, fatty acids rather than glucose are believed to be the main metabolic substrate for BAT (9). The effect of fatty meals on BAT metabolic activity is interesting, as fatty acids are presumed to be the physiologic activators of uncoupling protein 1 in rodents (9). Williams et al. compared the ${ }^{18} \mathrm{~F}-\mathrm{FDG}$ PET/CT scans of 741 patients who had taken a high-fat, very-lowcarbohydrate, protein-permitted meal with those of 1,229 patients who had fasted (11). The patients who had taken the high-fat meal had a significantly lower frequency of ${ }^{18} \mathrm{~F}$-FDG uptake in BAT and lower blood glucose levels than the patients who had fasted. Although this study was retrospective, it did support the findings of the present study.

It is tempting to speculate that BAT-mediated glucose clearance was responsible for the observed decreased postprandial glucose levels, compared with baseline levels. However, this speculation is not supported by our ${ }^{18} \mathrm{~F}-\mathrm{FDG}$ PET data. Moreover, lean and healthy subjects often have lower plasma glucose levels after ingestion of glucose than do obese volunteers (12). Whether in humans BAT functions as an organ that significantly contributes to substrate clearance, as has been shown for animals (13), remains to be established. The BAT-negative volunteers seemed to have lower postprandial insulin levels. These apparent differences in postprandial insulin levels were driven primarily by 1 patient with curiously low insulin levels after the meal.

\section{CONCLUSION}

We showed that cold-stimulated ${ }^{18} \mathrm{~F}-\mathrm{FDG}$ uptake by BAT in humans is more pronounced in the fasting state. The postprandial decrease in ${ }^{18} \mathrm{~F}$-FDG uptake by BAT may be explained by meal-induced insulin secretion or a switch to meal lipids as a substrate for BAT. Therefore, the contribution of carbohydrates and lipids as substrates to BAT activity during fasting and postprandial conditions in humans should be the subject of further study to confirm the role of BAT as a potentially important energy-dissipating organ.

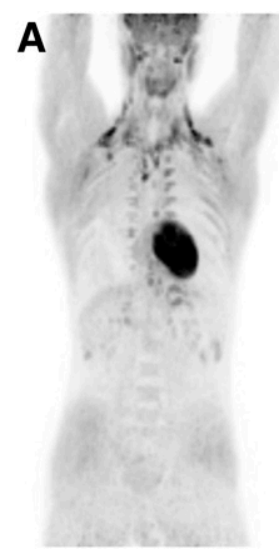

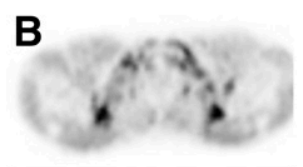

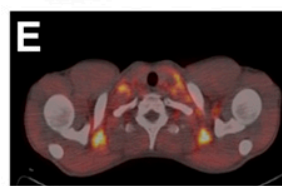

G

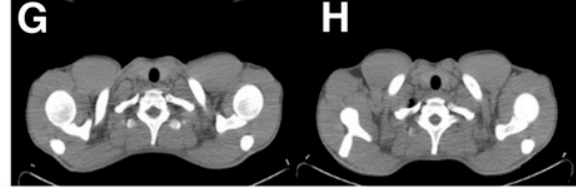

C

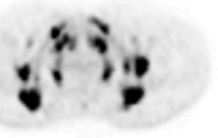

$\mathbf{F}$

Qhe

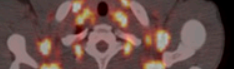

$2+\frac{1}{20} \rightarrow 0$

H

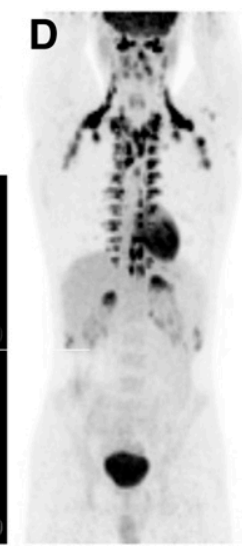

FIGURE 2. Impact of feeding on ${ }^{18} \mathrm{~F}-\mathrm{FDG}$ in BAT. Maximum-intensity projections show clear difference in ${ }^{18} \mathrm{~F}-\mathrm{FDG}$ between postprandial (A) and fasting (D) conditions. Transversal PET images show increased uptake in muscle and decreased uptake in BAT postprandially ( $\mathrm{B}, \mathrm{E}$, and $\mathrm{G})$, compared with during fasting $(C, F$, and $H)$. Uptake is superimposed on adipose tissue in correlated $C T$ images $(G$ and $H)$. $E$ and $F=$ $\mathrm{PET} / \mathrm{CT}$ images. 


\section{DISCLOSURE STATEMENT}

The costs of publication of this article were defrayed in part by the payment of page charges. Therefore, and solely to indicate this fact, this article is hereby marked "advertisement" in accordance with 18 USC section 1734.

\section{ACKNOWLEDGMENT}

No potential conflict of interest relevant to this article was reported.

\section{REFERENCES}

1. Cypess AM, Lehman S, Williams G, et al. Identification and importance of brown adipose tissue in adult humans. N Engl J Med. 2009;360:15091517 .

2. van Marken Lichtenbelt WD, Vanhommerig JW, Smulders NM, et al. Cold-activated brown adipose tissue in healthy men. $N$ Engl J Med. 2009;360: 1500-1508.

3. Virtanen KA, Lidell ME, Orava J, et al. Functional brown adipose tissue in healthy adults. $N$ Engl J Med. 2009;360:1518-1525.

4. Cypess AM, Kahn CR. Brown fat as a therapy for obesity and diabetes. Curr Opin Endocrinol Diabetes Obes. 2010;17:143-149.
5. Saito M, Okamatsu-Ogura Y, Matsushita M, et al. High incidence of metabolically active brown adipose tissue in healthy adult humans: effects of cold exposure and adiposity. Diabetes. 2009;58:1526-1531.

6. Vallerand AL, Lupien J, Bukowiecki LJ. Interactions of cold exposure and starvation on glucose tolerance and insulin response. Am J Physiol. 1983;245:E575E581.

7. Bukowiecki LJ. Energy balance and diabetes: the effects of cold exposure, exercise training, and diet composition on glucose tolerance and glucose metabolism in rat peripheral tissues. Can J Physiol Pharmacol. 1989;67: 382-393.

8. Orava J, Nuutila P, Lidell ME, et al. Different metabolic responses of human brown adipose tissue to activation by cold and insulin. Cell Metab. 2011;14:272279.

9. Marette A, Bukowiecki LJ. Noradrenaline stimulates glucose transport in rat brown adipocytes by activating thermogenesis: evidence that fatty acid activation of mitochondrial respiration enhances glucose transport. Biochem J. 1991;277: 119-124.

10. van Baak MA. Meal-induced activation of the sympathetic nervous system and its cardiovascular and thermogenic effects in man. Physiol Behav. 2008;94: $178-186$.

11. Williams G, Kolodny GM. Method for decreasing uptake of ${ }^{18}$ F-FDG by hypermetabolic brown adipose tissue on PET. AJR. 2008;190:1406-1409.

12. Soeters MR, Sauerwein HP, Faas L, et al. Effects of insulin on ketogenesis following fasting in lean and obese men. Obesity (Silver Spring). 2009;17: 1326-1331.

13. Bartelt A, Bruns OT, Reimer R, et al. Brown adipose tissue activity controls triglyceride clearance. Nat Med. 2011;17:200-205. 